\title{
A rare case of triplet heterotopic pregnancy with a live intrauterine and bilateral tubal ectopic
}

\author{
Poonam Agarwal $^{1 *}$, Kiran Raman Goyal ${ }^{1}$, Apurv Agarwal ${ }^{2}$
}

\begin{abstract}
${ }^{1}$ Department of Obstetrics and Gynecology, Sri Balaji Action Medical Institute, Delhi, India
${ }^{2}$ Medical Student, Maulana Azad Medical College, Delhi, India
\end{abstract}

Received: 26 May 2020

Accepted: 10 June 2020

\author{
*Correspondence: \\ Dr. Poonam Agarwal, \\ E-mail: poonam171166@gmail.com
}

Copyright: (c) the author(s), publisher and licensee Medip Academy. This is an open-access article distributed under the terms of the Creative Commons Attribution Non-Commercial License, which permits unrestricted non-commercial use, distribution, and reproduction in any medium, provided the original work is properly cited.

\begin{abstract}
Heterotopic pregnancy (HP) is a condition characterized by the coexistence of an ectopic pregnancy (EP) with a viable intrauterine pregnancy (IUP). The occurrence of a triplet heterotopic pregnancy is an exceptionally rare medical condition. Hence, timely diagnosis and management are challenging, but essential to prevent mortality. Authors report the case of a young woman who presented with a heterotopic triplet pregnancy, after in-vitro fertilization (IVF), at 12 weeks of gestation. She had been misdiagnosed as a case of severe ovarian hyperstimulation syndrome but had a ruptured tubal ectopic on the right side and an unruptured ectopic on the left side. Both the ectopics were managed by performing an emergency laparotomy with bilateral salpingectomy. The live intrauterine pregnancy was continued till term with the delivery of a healthy baby. High clinical suspicion and timely treatment can preserve the intrauterine gestation thus, ensuring a successful outcome.
\end{abstract}

Keywords: Ectopic pregnancy, Heterotopic pregnancy, In-vitro fertilization, Salpingectomy

\section{INTRODUCTION}

Heterotopic pregnancy (HP) is a rare condition in obstetrics, defined as the simultaneous presence of an intrauterine pregnancy (IUP) along with ectopic pregnancy (EP). It reportedly occurs in around 1 in 30000 spontaneous pregnancies. ${ }^{1,2}$ Incidence has been found to be greater in pregnancies after assisted reproductive technique (ART), ranging from $1: 100$ to $1: 3600$ and nearly as high as $1 \%$ in some series. ${ }^{1,2}$ Currently, the overall incidence of Heterotopic pregnancy is estimated at around $1: 7000$ to $1: 15000$ live births. ${ }^{3}$ Heterotopic triplets with a bilateral tubal ectopic pregnancy is a remarkably rare clinical scenario.

In a case report published by Fukuda et al, only 8 cases of bilateral tubal ectopic with IUP have been reported so far. ${ }^{4}$ Preservation of live IUP till term with a successful outcome is even rarer in such cases. The rate for achieving a viable pregnancy till term was found to be around $66 \%$ in a case series of 12 patients, while the remaining ended in miscarriage. ${ }^{5}$ In this article authors describe, a rare case of Heterotopic triplet pregnancy with bilateral tubal and intrauterine pregnancies after in-vitro fertilization (IVF), presenting with an acute abdomen due to rupture of one of the tubal ectopics. The objective of this article is to generate clinical awareness about this condition and aid in early diagnosis and timely treatment of such pregnancies.

\section{CASE REPORT}

A 31-year-old woman, G2P0A1, at 12 weeks of gestation presented at midnight with acute abdominal pain for one day. She had a fainting attack lasting for a few minutes on the previous day and vomiting for 5-6 days. She had conceived after IVF-ET with the transfer of 3 embryos and was referred with a probable diagnosis of severe ovarian hyperstimulation syndrome. She was under-going infertility treatment for 3 years with a diagnostic 
laparoscopy done 2 years back, which showed bilateral mild hydrosalpinx.

On admission, pulse rate was $110 / \mathrm{min}$, BP was $90 / 60$ $\mathrm{mmHg}$ along with severe pallor. The abdomen was distended, diffusely tender with rigidity and guarding, and a $\mathrm{Hb}$ of $7.1 \mathrm{~g} / \mathrm{dl}$. Resuscitation was done, and an urgent ultrasound showed a single live intrauterine gestational sac corresponding to 12 weeks, along with a large heteroechoic area and free fluid, suggestive of a ruptured right ectopic pregnancy (Figure 1). Transvaginal sonograms (TVS) done at 6 weeks and 8 weeks showed a single live intrauterine gestational sac corresponding to the gestational age without any adnexal mass. Blood was arranged, started, and the patient taken for emergency laparotomy with a probable diagnosis of heterotopic pregnancy. A right tubular ampullary ruptured ectopic pregnancy with blood and clots in the peritoneal cavity of around $1500 \mathrm{ml}$ were found intraoperatively. Right salpingectomy and evacuation of blood from the pelvis was performed with minimal manipulation of the uterus. On further exploration of the left adnexa, a mass of $2 \times 3 \mathrm{~cm}$ was seen at the is the ampullary junction on the left tube. Hence, a left salpingectomy was also performed (Figure 2). The patient was kept in ICU postoperatively, and 6 units of packed RBCs along with 4 units of FFP were transfused. On the 3rd postoperative day, a bedside ultrasound showed a live intrauterine pregnancy. The pregnancy was supported with progesterone as hydroxyprogesterone caproate 500 $\mathrm{mg}$ injection and dydrogesterone tablet $10 \mathrm{mg}$ TDS. The pathologist reported the presence of chorionic villi and trophoblastic tissue in both tubes, thus confirming the ectopic pregnancy.

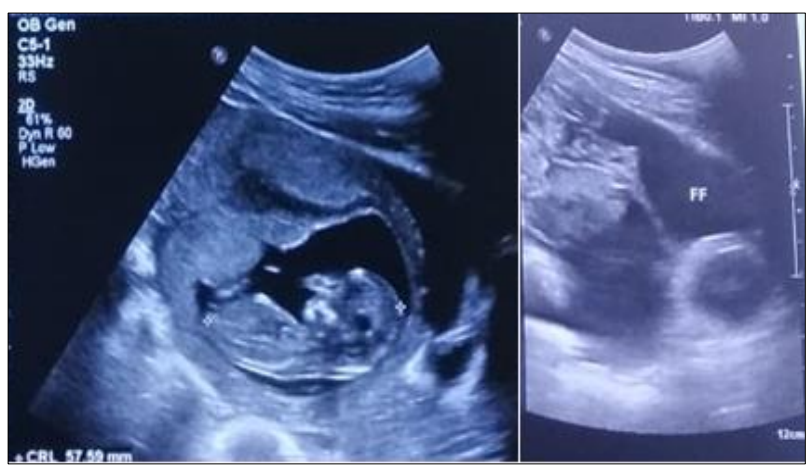

Figure 1: Ultrasound of intra-uterine pregnancy with moderate free fluid in abdomen.

Repeat ultrasound after 2 weeks showed a live intrauterine pregnancy of 15 weeks. At 18 weeks of gestation, the patient was admitted with pain lower abdomen and shortening of the cervix. Subsequently, McDonald's cervical cerclage was performed. At 26-27 weeks, she was admit-ted again with preterm labor. Two doses of injection betamethasone were given for fetal lung maturity and, tocolysis was achieved following which she was discharged. Finally, she was admitted at
37 weeks for an elective LSCS with the delivery of a live healthy female baby weighing $2.8 \mathrm{~kg}$.

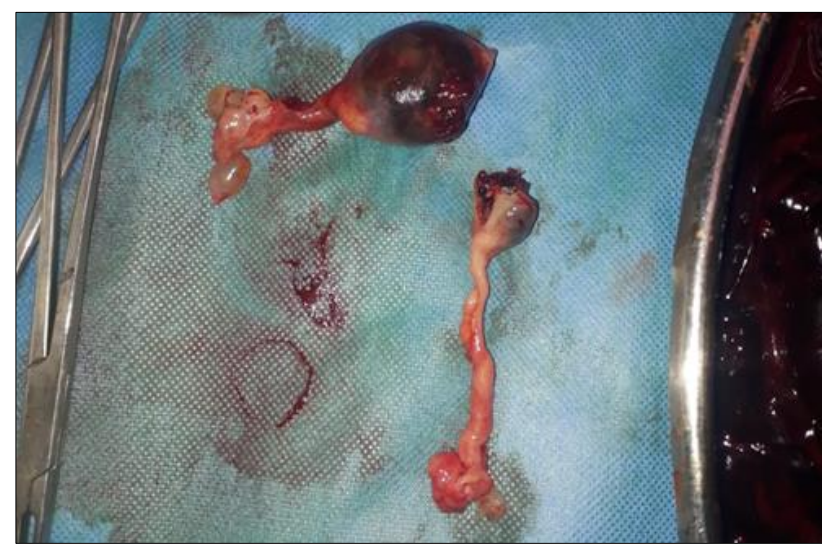

Figure 2: Specimen of right and left fallopian tubes of ectopic pregnancy.

\section{DISCUSSION}

Heterotopic triplets are rarely encountered in everyday obstetrics. Even in high-risk cases, the presence of live IUP is reassuring and as a result, the adnexa are often overlooked by the clinician as well as the radiologist. Hence, the patient usually presents in an acute emergency after a tubal rupture, and saving the life of the mother along with the intrauterine fetus becomes challenging. With the advent of ART, the incidence of HP has increased to $33: 10000$ after ovulation induction and 100:10000 after IVF. ${ }^{6}$ This could be attributed to varied factors, for instance, large amount of transferred embryos, a transfer near the uterine horn, excessive pressure on the syringe and deep insertion of the catheter during the transfer, the quality of embryos, the hormonal milieu at the instant of transfer, and the use of gonadotropins. $^{7}$ In women undergoing infertility treatment, additional factors such as the presence of PID, tubal damage due to causes like endometriosis, tuberculosis, and previous surgeries predispose them to ectopics pregnancies. While performing ART certain factors play a role in the incidence of ectopic pregnancy. The incidence of ectopic pregnancy has been reported to be significantly lower if embryo transfer is done on day 5 instead of day $3 .^{8}$ Additionally, it is lower for frozen blastocyst transfer, as compared to fresh blastocyst transfer. ${ }^{9}$ According to recent green guidelines, the ART process should be separated into 2 parts, create the embryos in the first month and transfer them after freezing in the second month. Recent studies have shown no correlation between the number of transferred embryos and hetero-topic pregnancy. ${ }^{6}$

Diagnosis of heterotopic pregnancy in a woman presenting with an acute abdomen is extremely challenging. In many cases, the patient is referred to the higher center with a provisional diagnosis of ovarian torsion/rupture, appendicitis, or ovarian hyperstimulation 
syndrome. In such cases, a high degree of clinical suspicion by the residents who are working in obstetrics emergencies is required. The principle diagnostic aid is transvaginal sonography (TVS), with a recent study by $\mathrm{Li}$ et al, demonstrating $92.4 \%$ sensitivity and $100 \%$ specificity for detecting HP. ${ }^{10}$ The role of se-rial $\beta \mathrm{HCG}$ is found to be limited due to coexisting viable IUP, which can generate an appropriate increase in the levels.

Currently, there is no consensus on the treatment of HP, and the majority of data about its clinical management is derived from case reports. The management is aimed at removing the ectopic pregnancy without disturbing the viable IUP. Various conservative treatment options like the injection of feticide under ultrasound guidance and expectant management have been reported but, the gold standard is surgery via laparotomy or laparoscopy depending on the clinical scenario. ${ }^{11}$ A thorough abdominal and pelvic inspection must be done during surgery for an ectopic gestation, especially on the contralateral side. Frequently, the contralateral pregnancy is discovered a few days to weeks after the initial surgery. The prognosis of intrauterine gestation after early treatment of the coexisting ectopic is favorable.

\section{CONCLUSION}

Early diagnosis and prompt intervention are crucial to diminish the morbidity and mortality of HP. Considering the increased risk of HP after IVF-ET, vigilant follow up of women with a positive pregnancy test is recommended throughout early pregnancy. Performing a thorough TVS be-tween 4 to 6 weeks of gestation could allow early detection and successful conservative management.

Funding: No funding sources

Conflict of interest: None declared

Ethical approval: Not required

\section{REFERENCES}

1. Basile F, Di Cesare C, Quagliozzi L, Donati L, Bracaglia M, Caruso A, et al. Spontaneous heterotopic pregnancy, simultaneous ovarian, and intrauterine: a case report. Case Reports Obstet Gynecol. 2012;2012:1-4.

2. Felekis T, Akrivis C, Tsirkas P, Korkontzelos I. Heterotopic triplet pregnancy after in vitro fertilization with favorable outcome of the intrauterine twin pregnancy subsequent to surgical treatment of the tubal pregnancy. Case Reports Obstet Gynecol. 2014;2014:1-4.

3. Selo-Ojeme D, GoodFellow C. Simultaneous intrauterine and ovarian pregnancy following treatment with clomiphene citrate. Arch Gynecol Obstet. 2002;266(4):232-4.

4. Fukuda T, Inoue H, Toyama Y, Ichida T, Uzawa Y, Monma $\mathrm{M}$, et al. Bilateral tubal and intrauterine pregnancies diagnosed at laparoscopy. Journal of Obstet Gynaecol Res. 2014;40(10):2114-7.

5. Luo X, Lim C, Huang C, Wu J, Wong W, Cheng N. Heterotopic pregnancy following in vitro fertilization and embryo transfer: 12 cases report. Arch Gynecol Obstet. 2009;280(2):325-9.

6. Jeon J, Hwang Y, Shin I, Park C, Yang K, Kim H. The risk factors and pregnancy outcomes of 48 cases of heterotopic pregnancy from a single center. J Korean Med Sci. 2016;31(7):1094.

7. Divry V, Hadj S, Bordes A, Genod A, Salle B. Case of progressive intrauterine twin pregnancy after surgical treatment of cornual pregnancy. Fertil Steril. 2007;87(1):190.e1-190.e3.

8. Zhang B, Cui L, Tang R, Ding L, Yan L, Chen Z. Reduced ectopic pregnancy rate on day 5 embryo transfer compared with day 3: a meta-analysis. Plos One. 2017;12(1):e0169837.

9. Li Z, Sullivan E, Chapman M, Farquhar C, Wang Y. Risk of ectopic pregnancy lowest with transfer of single frozen blastocyst. Human Reprod. 2015;30(9):2048-54.

10. Li X, Ouyang Y, Lu G. Value of transvaginal sonography in diagnosing heterotopic pregnancy afterin-vitro fertilization with embryo transfer. Ultrasound Obstet Gynecol. 2013;41(5):563-9.

11. Arsala L, Danso D. Spontaneous heterotopic triplet pregnancy with tubal rupture. J Invest Med High Impact Case Rep. 2014;2(2):232470961453155.

Cite this article as: Agarwal P, Goyal KR, Agarwal A. A rare case of triplet heterotopic pregnancy with a live intrauterine and bilateral tubal ectopic. Int $\mathrm{J}$

Reprod Contracept Obstet Gynecol 2020;9:3087-9. 